\title{
TerraSAR-X Antenna Calibration and Monitoring Based on a Precise Antenna Model
}

\author{
Markus Bachmann, Marco Schwerdt, Benjamin Bräutigam
}

\begin{abstract}
The high flexibility and tight accuracy requirements of modern spaceborne SAR systems require innovative technologies to calibrate and process the SAR images. To perform accurate pattern correction during SAR processing, an antenna model can be used to derive the multitude of different antenna beams generated by active antenna steering. The application of such an antenna model could be successfully demonstrated for the TerraSAR-X mission, launched in 2007. The methodology and the results of the in-orbit verification with an achieved accuracy of better than $\pm 0.2 \mathrm{~dB}$ is reviewed in this paper in detail showing its outstanding accuracy. Additionally, the results of the antenna pattern long term monitoring are described pointing out the high stability of the system
\end{abstract}

Index Terms-Active antenna calibration, antenna modelling, TerraSAR-X calibration

\section{Motivation}

The accurate knowledge of the antenna patterns of a synthetic aperture radar (SAR) is of main importance for precise SAR image processing. The antenna patterns are required to correct the antenna characteristics visible in the image, as shown in Figure 1. On the left, the uncorrected image shows a high brightness in the centre and a decreasing illumination to the borders of the image in range direction. This is caused by the antenna pattern spanning over range. The right image presents the same acquisition after antenna pattern correction with the characteristics of the antenna being eliminated.

Early SAR systems like ERS1/2 or XSAR/SRTM with a low number of antenna beams used in-orbit antenna pattern measurements for correction. The Envisat/ASAR instrument for example acquires SAR images with only eight different antenna beams. Although already at ASAR an antenna model was implemented, the more accurate antenna patterns were obtained from in-orbit measurements. Images over homogeneously distributed targets were used to determine the reference patterns for each individual beam [1].

Manuscript received February 27, 2009; revised July 28, 2009; September 9, 2009. Current version published September 28, 2009.

The authors are with the Microwaves and Radar Institute, German Aerospace Center, 82234 Wessling, Germany (e-mail: markus.bachmann@dlr.de; marco.schwerdt@dlr.de; benjamin.braeutigam@dlr.de).

Color versions of one or more of the figures in this paper are available online at http://ieeexplore.ieee.org.

Digital Object Identifier TGRS-2009-00149
In contrast to these systems, actual SAR satellites enable a very high number of different acquisition modes like Stripmap, ScanSAR or other wide swath and high resolution modes. Thus, a multitude of different antenna beams and hence antenna patterns are needed for consistent calibration of the modes against each other and within the SAR image itself. Enabling object detection and classification novel SAR systems have very tight accuracy requirements. They produce images with high resolution in meter-range and accurate measures of the backscatter down to a few tenth of $\mathrm{dB}$.

A further important point for satellite calibration is the duration of the calibration process, the commissioning phase of the satellite in space. Current SAR systems are no longer experimental systems designed for scientific experiments which have uncritical schedule constraints. In fact they shall be used for commercial applications to a great extend. Driven by paying customers, the system has to be available as early as possible or at least at an exactly determined date. Hence, a short duration of the commissioning of the satellite is evident.

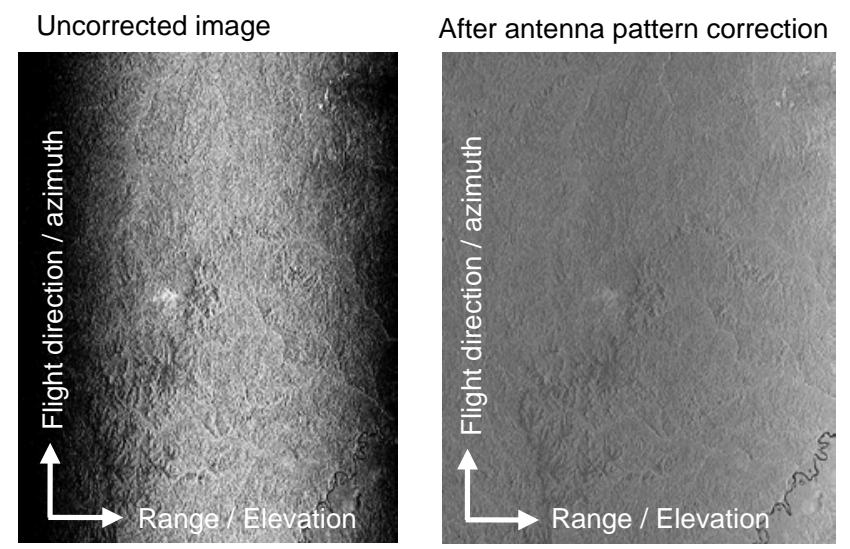

Figure 1: SAR image before and after pattern correction

Reviewing these points, innovative methods are necessary to calibrate complex SAR systems. In this context, the most important key element is the antenna model approach described below. It derives the antenna patterns from mathematical models in combination with on-ground measurements characterising parts of the array antenna. With this approach it becomes possible to calibrate a high number of used antenna beams not only with high accuracy but also very time and cost effective. This is demonstrated in the example of the TerraSAR-X system. 


\section{INTRODUCTION ON THE TERRASAR-X SYSTEM}

The TerraSAR-X satellite [2], launched in June 2007, is a flexible X-Band SAR satellite built in a Public Private Partnership (PPP) between the German Aerospace Center (DLR) and Astrium GmbH. The main payload of TerraSAR-X is a SAR instrument for the acquisition of high quality radar images of the Earth's surface.

The SAR instrument comprises an active phased array antenna which allows flexible beam forming with a centre frequency of $9.65 \mathrm{GHz}$ and a maximal bandwidth of $300 \mathrm{MHz}$. The antenna with its length of $4.8 \mathrm{~m}$ and its width of $0.7 \mathrm{~m}$ consists of 384 subarrays composed by two slotted wave-guides, one for each polarisation (horizontal and vertical). These are arranged in 12 panels in azimuth direction (columns) each composed of 32 subarrays (rows) [3]. The nominal antenna pointing in elevation is $33.8^{\circ}$ away from nadir, the full performance range covers incidence angles from $20 \mathrm{deg}$ and $40 \mathrm{deg}$ and the allowed steering range is from -20 deg to +20 deg of antenna look angle. Right and left looking acquisition is realised by satellite roll manoeuvres.

Each individual subarray is driven by a Transmit/Receive Module (TRM) adjustable in amplitude and phase by applying complex excitation coefficients. This enables beam steering and adaptive beam forming in both azimuth and elevation direction.

More than 12000 different beams can be commanded for the multitude of standard acquisition modes possible on TerraSAR-X [2]. These are the nominal Stripmap, ScanSAR or Spotlight modes as well as several experimental modes like quad-polarisation mode, along-track interferometry or the novel TOPS mode [15].

The accuracy requirement goal for the overall radiometric accuracy on TerraSAR-X is $1.0 \mathrm{~dB}$ ( 1 sigma). This value was derived from the radiometric error budget calculated prior to the development of TerraSAR-X. All radiometric errors affecting the SAR acquisition were considered in this error budget. The main contributions are the accuracy of the internal calibration, the error of the antenna model, the mechanical and electrical antenna pattern variation, processing errors, the accuracy and stability of the measurement targets and atmospheric variations. In this budget, the requirement for the accuracy of the antenna model is to be better than $\pm 0.2 \mathrm{~dB}$ (peak-to-peak) for reproducing the pattern shape and predicting the gain offset between different beams. Another important reason for this number is that a deviation of more than $0.2 \mathrm{~dB}$ can visibly be recognized in overlaid or adjacent SAR images.

\section{ANTENNA MODEL APPROACH}

\section{A. Purpose of the antenna model}

The antenna model is used to compute the huge amount of antenna patterns needed by the processing system to correct the impact of the antenna characteristics on the radar images. In elevation, the antenna patterns are then used for direct image correction over range. The azimuth pattern is represented in the Doppler spectrum and hence needed for correct Doppler estimation.

A second important task than can be realised with the antenna model is the optimisation of the beam excitation coefficients of the antenna array. These excitation coefficients are complex values which are applied on the TRMs in order to steer the beam in the desired direction as well as to create an antenna pattern with an optimised gain, a desired pattern slope or suppressed side-lobes. During the optimisation process the excitation coefficients are varied and the resulting pattern is calculated until they converge against pre-defined quality parameters. With this optimisation process, an optimum set of excitation coefficients and hence an optimum performance for the full performance beams in terms of Noise Equivalent Sigma Zero (NESZ) and Total Ambiguity Ratio (TAR) is achieved.

Also, in case of contingences like TRM degradation or failures during the operational phase resulting in a degradation of the antenna patterns, the antenna excitation coefficients can be re-optimized to ensure the high performance [13].

The development and the establishment of an antenna model approach were driven by three main requirements:

- The large number of more than 12000 different beams to be calibrated

- The tight accuracy requirement of an overall radiometric accuracy of better than $1.0 \mathrm{~dB}$ (1 sigma) as explained in Chapter II.

- The short duration of the commissioning phase of less than six months.

\section{B. Quality control}

To ensure these requirements, several steps were realized.

As much effort as possible was moved from in-orbit tasks to on-ground duties. This includes the accurate measurement of the embedded subarray patterns as well as the validation of the model on-ground before launch. The task was successfully performed by Astrium and DLR, and is described in more detail in chapter $\mathrm{V}$.

Different in-orbit calibration techniques for antenna model verification were used. Therefore, in-orbit verification was performed during the commissioning phase in the first months after launch. The verification was divided into three tasks, which are described in detail in Chapter VI:

- measurements across the rainforest to verify the elevation pattern shape

- the use of ground receivers to verify the azimuth pattern and

- ScanSAR measurements over rainforest and over ground receivers to verify the prediction of the gain offset between the beams.

To ensure a short commissioning phase, the verified antenna model supported the absolute radiometric calibration which is the determination of the absolute calibration factor. 
With a verified antenna model, the absolute calibration factor of only one beam needs to be determined which significantly minimizes the effort for the absolute radiometric calibration. For verification purpose, several few representative beams were selected to verify the calibration approach. A description of the absolute radiometric calibration would exceed the content of this paper and the interested reader is referred to [7] and [9].

The described approach is summarized in Figure 2. The starting point is the design of the antenna model. This is the definition of the algorithms as well as the implementation of the pattern synthesis and pattern optimisation. Therefore, the subarray patterns are needed as an input. Then, the model is validated on ground first, using validation patterns measured on whole panels. After launch, the verification of the model is performed by in-flight measurements over distributed targets like rainforest and point targets like ground receivers. Hereby the actual state of the instrument and the TRMs is monitored simultaneously by applying the Internal Calibration facility and the so called PN gating method in the instrument [13].

The Internal Calibration measures the actual state of transmit and receive path within the radar instrument, which afterwards can be corrected during image processing. It is performed at every beginning and end of an acquisition.

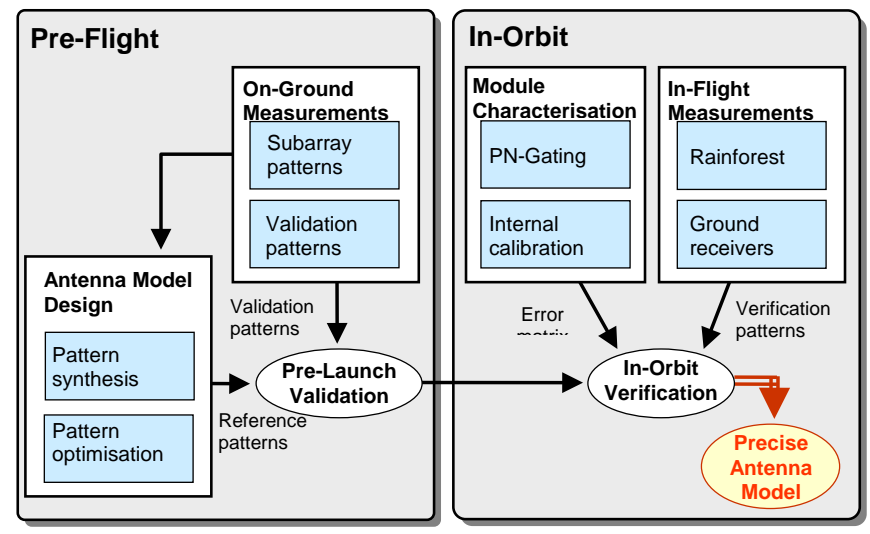

Figure 2: Antenna model verification approach

\section{The PN-Gating method}

The PN-Gating method [13] is a novel approach to monitor the actual state of each individual TRM. It was demonstrated on TerraSAR-X for the first time in-orbit. In contrast to the module stepping approach applied on Envisat/ASAR where each TRM was measured sequentially, the TRMs on TerraSAR-X are characterised during quasi-nominal operation that means with all TRMs enabled. Hence, the TRMs are driven under most realistic conditions while they are characterised.

Due to the different paths through the instrument for transmit and receive, calibration pulses are routed through the instrument in both directions separately in order to separate the influence of high power amplifiers and low noise amplifiers. This is realised by applying orthogonal pseudo noise (PN) or Walsh codes to each individual TRMs. The measured sum signal can be correlated with the individual codes to extract the single gain and phase states of the TRMs.

The PN-Gating method is applied regularly to detect changes, drifts or failing TRMs. If the changes exceed a given limit, the antenna patterns have to be re-calculated or even reoptimised. The method was verified on ground where the deactivation of one or several TRMs could be clearly detected. Results of the long term stability of the TRMs show a high stability and are shown in chapter VI.A.

With this antenna model sufficiently validated on ground and verified by a limited number of a few selected beams really measured in-flight, the thousands of reference patterns can now be accurately derived.

\section{Antenna Model Design}

The antenna model itself mathematically calculates radiation patterns by the superposition of four inputs:

- radiation patterns measured on ground from the array elements of the antenna, so called embedded subarray patterns,

- beam excitation coefficients (amplitude and phase) of each individual transmit/receive module (TRM),

- exact geometrical dimensions of the array antenna including the distances between the subarrays,

- the actual state of the SAR instrument like drifting and/or failed TRMs.

For active phased array antennas, the radiated pattern $F_{\text {Beam }}$ is calculated by [3], [12]:

$$
\begin{aligned}
\vec{F}_{\text {Beam }}(\varepsilon, \alpha)=\sum_{m=0}^{M-1} \sum_{n=0}^{N-1}\left(\vec{C}_{S A, m n}(\varepsilon, \alpha) \cdot a_{m n} \cdot E_{S A, m n} \cdot\right. \\
\left.e^{j k \sin \varepsilon \cos \alpha\left(-\frac{N-1}{2}+n\right) \Delta y} \cdot e^{j k \cos \varepsilon \sin \alpha\left(-\frac{M-1}{2}+m\right) \Delta x}\right)
\end{aligned}
$$

with the desired elevation and azimuth angle $\varepsilon$ and $\alpha$, the amount of subarrays $N$ rows and $M$ columns, the intersubarray distances $\Delta x$ (columns) and $\Delta y$ (rows). The wave number $k$ includes the centre frequency $9.65 \mathrm{GHz}$ of the system by the relation of $k=2 \pi / \lambda$.

The embedded subarray patterns $C_{S A}$ comprise the real measured radiation characteristics of the individual subarray elements. The subarrays are embedded into the whole antenna and have to be given for each row, column, elevation and azimuth angle. The embedded pattern of one subarray mounted in the array antenna describes the radiation characteristic of this subarray. In this way, mutual coupling effects are included by the measurements. The antenna has a broad frequency bandwidth of $300 \mathrm{MHz}$. However, the nominal full performance modes utilise only $150 \mathrm{MHz}$ or $100 \mathrm{MHz}$ during acquisition, $300 \mathrm{MHz}$ bandwidth is only for experimental modes. For the nominal acquisition, mismatch and gain are smooth over the frequency bandwidth. Therefore, 
only the embedded patterns at centre frequency are incorporated in the antenna model. The validity of this approach is verified with the calibration approach as shown in chapter VI.

As input for the antenna model, all embedded subarrays of one panel, which is 32 subarrays in a column, were measured. The patterns of whole panels are very similar to each other, independent from the position of the panel which means if it is situated in the centre of the leaf or at its edges. The measurement results showed that it is possible to use only the embedded patterns of one panel and substitute the others using their measured amplitude and phase offsets. These embedded patterns have to be known with high accuracy, as they are one main input of the model. The measurements have to be at least more accurate than the required setting accuracy of the TRMs, that is $0.5 \mathrm{~dB}$ and $5 \mathrm{deg}$ of phase.

The commanded complex excitation coefficients are given by $a$. In case of TerraSAR-X, these coefficients are provided in terms of amplitude and phase values row and column-wise. For each commandable beam, one set of values is put in a common table. This table is available on board of the satellite and can be updated if necessary, e. g. in case of contingencies, as the on-board computer uses the table for each acquisition.

Finally, the error matrix $E_{S A}$ describes drifting or failed antenna elements. These are determined via the PN-Gating method using orthogonal code sequences applied to the TRMs as described in chapter III.

To obtain the complete two-way antenna patterns, the equation is evaluated for transmit and receive separately. Both, the excitation laws and the error matrix are different for transmit and receive. In case of the excitation coefficients, the differentiation allows greater flexibility for beam steering. On the side of the error matrix the radar signals travel through the TRMs on different paths, for transmit via the high power amplifier and in receive through the low noise amplifier.

For the TerraSAR-X SAR applications, cuts of the patterns in elevation (at $\alpha=0 \mathrm{deg}$ ) and azimuth ( $\varepsilon=0 \mathrm{deg}$ ) are derived. These are saved into interface tables to be applied for image correction in the SAR processor.

The accuracy of the antenna model is on the one hand mainly determined by the accurately measured embedded subarray patterns and on the other hand by the stability of the instrument. This includes the accurate measurement of the error matrix via PN-Gating as well as the correction of internal variations by the Internal Calibration. To prove the accuracy, sensitive in-orbit measurement methods are required as described below.

\section{Pre-Launch Validation}

After design and implementation of the antenna model, at first an on-ground validation has been performed. The onground characterisation was realised in two stages. In a first step, the antenna was accurately measured in the Planar Near Field Scanner at and from Astrium GmbH, Germany. In the second step, the correct application of the conventions and input parameters was verified.

For the first step, two kinds of antenna patterns were determined in the Planar Near Field Scanner:

The embedded subarray patterns are required as a direct input into the antenna model.

For comparison, the patterns of one complete panel or leaf (one third of the whole antenna) was measured.

Due to the dimensions of the whole antenna $(4.8 \mathrm{~m} \times 0.7 \mathrm{~m})$, the pre-launch validation was performed only up to the stage of one leaf, this is one third of the antenna or four panels in a column. To ensure a valid model even for the complete antenna, the accuracy of the antenna model was tracked through the whole built process of the antenna from individual subarray patterns over the patterns of one panel up to the patterns of the three leaves. Consistent accordance has been found within this development process between the antenna model and the measurements.

The measurement in an anechoic chamber is not exactly the same as if the antenna is mounted on the satellite. Although, as there are no parts of the satellite structure rising into the near field of the SAR Antenna, the effects of the satellite and its mounting structure were expected to be small. Additionally, the measurements on satellite level in orbit were expected to be better than the leaf-level measurements as three times more TRMs on the complete antenna provide better statistical distribution for the variation of the TRMs.

\begin{tabular}{|l|l|l|}
\hline Beam & \multicolumn{2}{|l|}{$\begin{array}{l}\text { Maximal Deviation within } \\
\text { in the 3 dB main lobe }\end{array}$} \\
\hline & $\mathrm{HH}$ & VV \\
\hline strip_003 & $+0.05 \mathrm{~dB}$ & $+0.15 \mathrm{~dB}$ \\
\hline strip_004 & $+0.10 \mathrm{~dB}$ & $+0.08 \mathrm{~dB}$ \\
\hline strip_005 & $+0.03 \mathrm{~dB}$ & $+0.14 \mathrm{~dB}$ \\
\hline strip_006 & $+0.08 \mathrm{~dB}$ & $+0.08 \mathrm{~dB}$ \\
\hline strip_007 & $+0.13 \mathrm{~dB}$ & $+0.04 \mathrm{~dB}$ \\
\hline strip_008 & $-0.06 \mathrm{~dB}$ & $-0.15 \mathrm{~dB}$ \\
\hline strip_009 & $-0.12 \mathrm{~dB}$ & $+0.10 \mathrm{~dB}$ \\
\hline strip_010 & $+0.10 \mathrm{~dB}$ & $+0.17 \mathrm{~dB}$ \\
\hline strip_011 & $+0.08 \mathrm{~dB}$ & $+0.09 \mathrm{~dB}$ \\
\hline strip_012 & $+0.09 \mathrm{~dB}$ & $+0.05 \mathrm{~dB}$ \\
\hline strip_013 & $+0.02 \mathrm{~dB}$ & $+0.07 \mathrm{~dB}$ \\
\hline strip_014 & $+0.11 \mathrm{~dB}$ & $+0.08 \mathrm{~dB}$ \\
\hline
\end{tabular}

Table 1: Results of the pre-launch validation in elevation. Deviation between measurement and antenna model.

For the pre-launch validation, all patterns were measured for both polarisation and at five frequencies. After the measurement, the obtained near field patterns were transformed into the far field using a Fourier transform algorithm. Then, the antenna patterns generated with the antenna model using the embedded patterns were compared to the measured patterns of the complete panel and leaf respectively. The results of this validation on leaf level exemplary for the centre leaf are depicted in Table 1 . The 
given values state the maximal deviation between simulated and measured antenna pattern within the $3 \mathrm{~dB}$ main lobe of the beam. It can be seen, that the deviation fulfils the required limit of $\pm 0.2 \mathrm{~dB}$ for all beams [5].

After the successful on-ground validation of the antenna model, the correct application of the conventions in the antenna control unit was verified on satellite level. Herefore the complete antenna was already mounted on and connected to the satellite system. Especially the correct handling of the underlying inputs like antenna excitation coefficients, the correct numbering of the antenna elements and the correct steering angle application were successfully tested. Also, the correct update of all changeable antenna parameters like TRM enabling/disabling or excitation coefficients table was verified.

The pre-flight validation proved a very stable and accurate instrument, followed by the successful in-orbit verification as shown in the next chapter.

\section{IN-ORBIT VERIFICATION}

The in-orbit verification of the antenna model was performed in the commissioning phase during the first six months after launch in the second half of the year 2007.

This chapter is divided into three sub-chapters. The first one describes the evaluation techniques used for antenna model verification, second showing the determination of the antenna pointing results and the last one providing detailed explanation of the model verification.

\section{A. Measurement evaluation techniques}

\section{1) Elevation pattern evaluation technique}

To verify the antenna model in elevation, again like on ground, the simulated relative antenna patterns are compared with measured antenna patterns. The estimates are derived from SAR images acquired over rainforest in the Amazon basin, Brazil. Amazon rainforest is in general a homogeneous scatterer [11] (see Figure 3a) and the pattern shape is clearly visible in the uncorrected SAR image data (compare Figure 1).

The correction of this effect is performed in the SAR processing chain. There, the accurate position and geometry of the acquisition is determined and annotated after azimuth and range compression. Then the antenna patterns mapped in the image data are corrected with the available modelled reference antenna patterns [6].

Processed SAR-Products in Single Look Slant Range geometry are used for pattern estimation. Thus, the applied antenna pattern correction has to be reversed with the used reference patterns to obtain the original impact of the antenna characteristics on the image.

Afterwards, the image is freed from disturbances like rivers using an automatic masking algorithm. This is depicted in
Figure 3, where the red/white pixels describe masked pixels that are not taken into account for pattern evaluation.

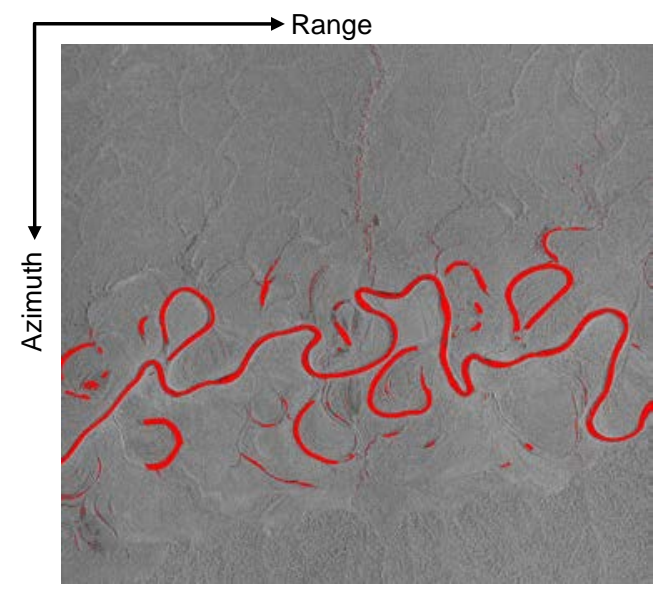

Figure 3: Exemplary rainforest test site

The processed radar image is denoted in beta nought $\beta_{0}$ where the backscatter depends on the incidence angle $\theta$ on the earth. For the comparison however, the gamma nought $\gamma_{0}$ has to be derived to omit the incidence angle dependency of the backscatter of the rainforest. Gamma nought is obtained via the sigma nought $\sigma_{0}$ using the formula [8]:

$$
\gamma^{0}=\sigma^{0} / \cos (\theta)=\beta^{0} \cdot \tan (\theta)
$$

Finally, the values of all azimuth lines are summed up and each pixel position is transformed as a function of the elevation angle resulting in a so called Gamma Profile which is a vector of the mean antenna pattern over elevation angle (compare Figure 8).

\section{2) Azimuth pattern evaluation technique}

In addition to elevation, also the antenna patterns in flight direction have to be verified. The verification of these azimuth antenna pattern was performed for transmit pattern using the DLR ground receivers [10] as depicted in Figure 4. In contrast to the elevation pattern, where only the main lobe is measured, also several side lobes can be determined in azimuth.

The ground receivers record the amplitudes of the pulses transmitted by the SAR antenna as function of time. Due to the flight movement, a cut through the antenna pattern is recorded. Transformed to the antenna azimuth angles and corrected by position information, the azimuth antenna pattern is obtained and can be compared to the modelled pattern.

The measurements were performed for patterns over the whole specified angular range of the SAR antenna, i.e. for low, mid and high incidence angles. Furthermore ground receivers were placed across each swath measuring at near, mid and far range. Mostly two receivers were deployed at one location, aligned for different polarisations. The locations were chosen such that no trees, buildings or mountains are near by to minimize multi-path effects. Also, the primarily measured "full performance" beams cover incidence angles 
between 20 deg and 45 deg. Hence, multi-path signals from the ground are reduced by the receiver's antenna pattern.

Unlike the elevation pattern measurement, the operation of ground receivers required much more man power. The receivers have to be placed to different locations for the different beams and they have to be aligned for each measurement.

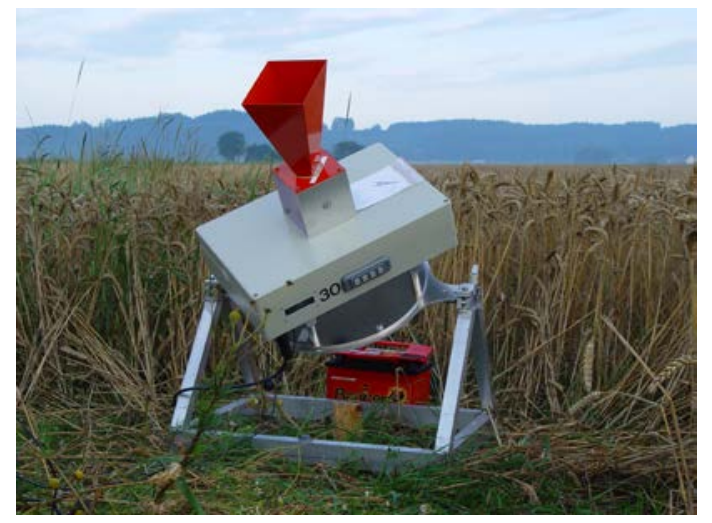

Figure 4: DLR Ground Receiver deployed for measurement
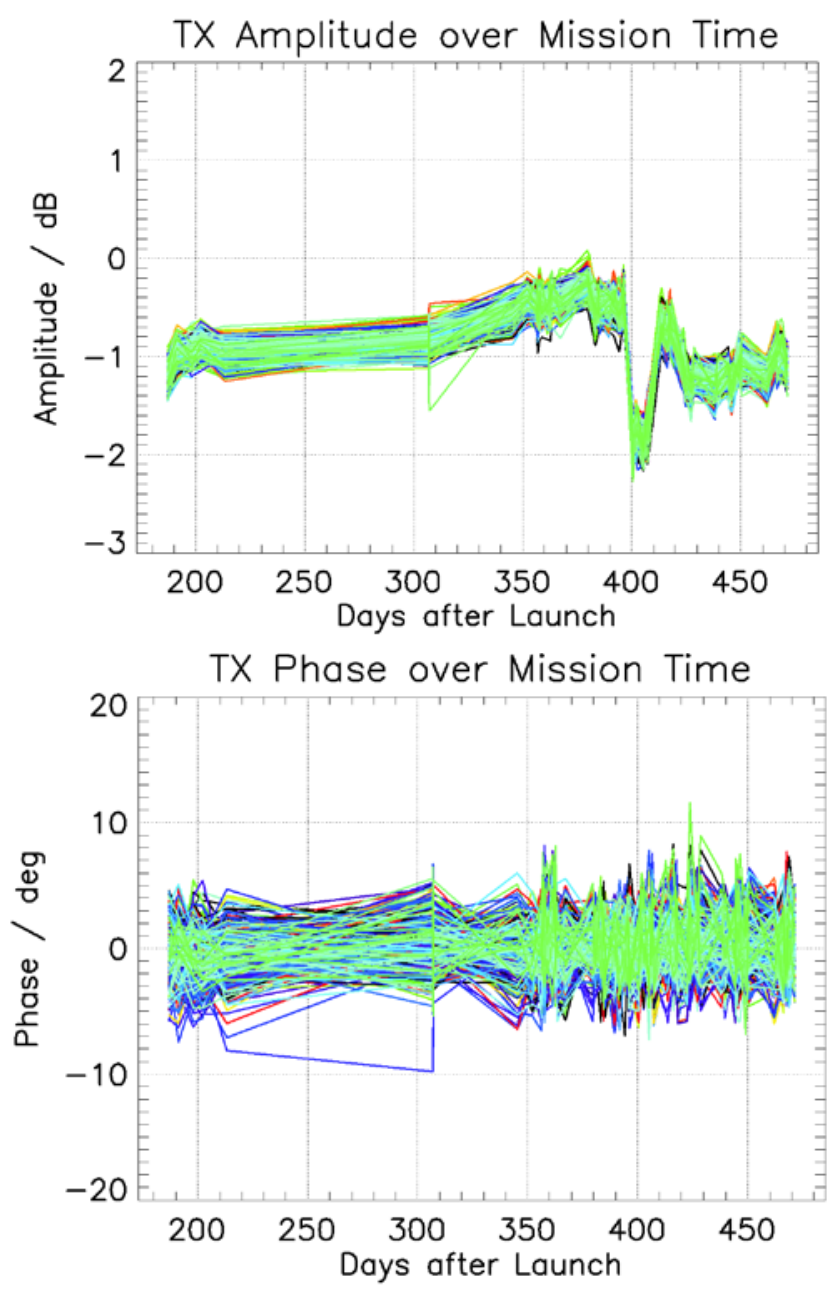

Figure 5a: Measurement variation of all TRMs transmit gains over time since start of the operational phase.

Figure 5b: Measurement variation of all TRMs transmit phase over time since start of the operational phase.

\section{B. Measurement of actual excitation coefficients}

To ensure a properly working SAR instrument, the individual state of the antenna excitation coefficients has to be checked prior to calibration. It is read from the actual TRM behaviour being realised by the PN Gating method used to characterise the active front-end modules. During commissioning phase, initial data sets have been acquired to compare the TRM in-orbit status to its start values. This was done during the commissioning phase and also later for long term monitoring being referenced to this initial data.

Figure 5 presents the TRMs' transmit long-term performance. The individual measurements are referenced to the respective average value determined during the satellite commissioning phase. The measurements show a stable behaviour over time with no TRM degradation or failure since launch. There is no trend for strong deviations compared to the reference values.

The TRMs' total gain variation over time is due to the different temperature conditions in the instrument. As this variation of the total instrument gain is likewise extracted from the calibration pulses and compensated for during ground processing, the system stays absolutely calibrated, as shown in chapter VII.

The above results on individual TRM performance prove the high stability of the TerraSAR-X active antenna of better than $0.2 \mathrm{~dB}$ (rms) in gain and $2^{\circ}$ (rms) in phase. These measurements also verify the successful implementation of the PN Gating method in a spaceborne environment for the first time ever.

\section{Pointing determination}

The first step for an accurate in-orbit verification is the determination of beam pointing errors. Pointing errors can result from mechanical and electrical antenna mis-pointing as well as attitude control offsets in the satellite.

For the pointing determination, a so-called notch pattern is applied to the SAR antenna. The resulting antenna pattern has a sharp slope at the boresight angle (compare Figure 6a). To obtain the notch, one half of the antenna is excited with a phase-difference of $180 \mathrm{deg}$ against the other half. Thus, the radiated signal cancels out in boresight direction. The measured offset of the notch compared to a reference pattern then directly provides the mis-pointing of the antenna.

\section{1) Elevation pointing determination}

The pointing determination in elevation was performed over rainforest as explained before. As the signal to noise ratio in rainforest acquisitions is below $20 \mathrm{~dB}$, the notch was applied in transmit direction only. Thus, a better comparison is possible as the notch in the reference pattern is not as deep as for a case with a notch in transmit and receive direction.

An example of the determination of the beam pointing in elevation performed on TerraSAR-X is shown in Figure 6a. Several measurements of eight different passes are shown in different colours. The different measurements have a very 
high accordance in terms of the position of the notch. The variation of the notch in the different measurements is less than $0.008 \mathrm{deg}$ which is half the required pointing determination accuracy.

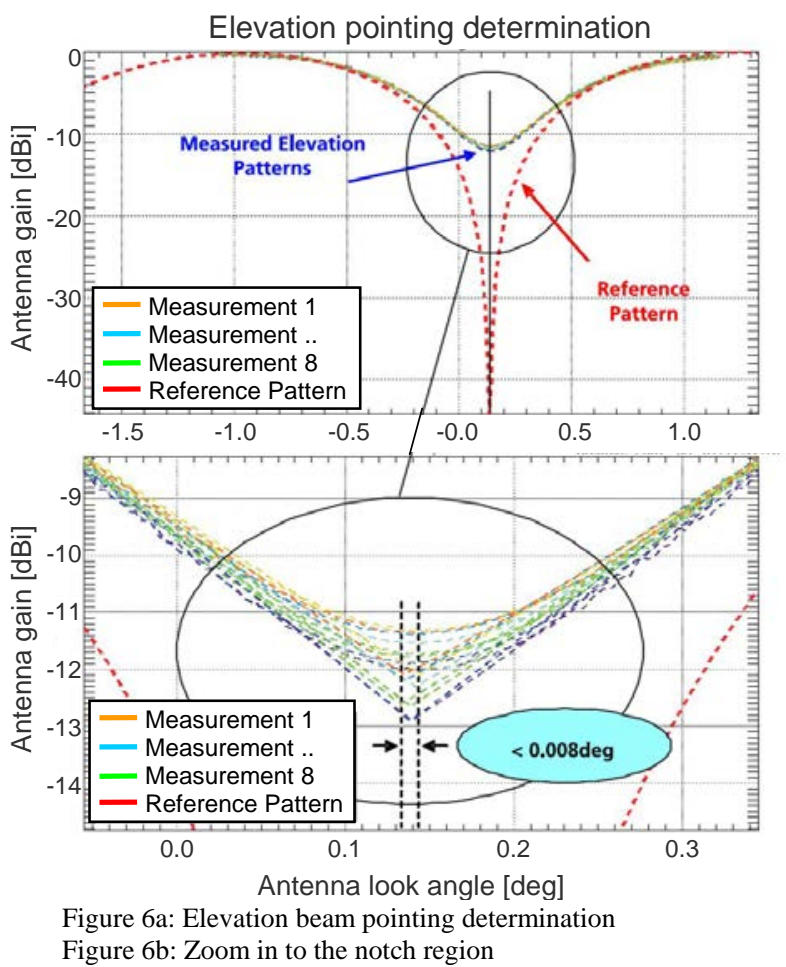

For TerraSAR-X, first a mis-pointing of $0.15 \mathrm{deg}$ in elevation was found. The reason was not finally clarified but is probably caused by small deviations in the star tracker alignments. It could be corrected updating the star tracker alignments in the satellite on-board computer. A residual deviation between $\mathrm{H}$ - and $\mathrm{V}$-polarisation of -0.01 deg for $\mathrm{H}$ and +0.02 deg for $\mathrm{V}$-polarisation was corrected directly in the reference antenna patterns for the SAR processor.

\section{2) Azimuth pointing determination}

In azimuth, only the pointing in transmit direction can be evaluated. Though, due to the reciprocity of the wave guides and the knowledge of the active part of the antenna by PNGating, the pointing is assumed to be the same also in receive direction.

For the pointing determination, the exact timing information is of fundamental importance. From the timing in combination with the accurate orbit information, the real as well as the assumed position of the notch can be calculated transforming the orbital state vectors in the antenna coordinate system.

Using the notch pattern, for TerraSAR-X a pointing knowledge better than 0.002 deg or $16 \mathrm{~Hz}$ of Doppler has been achieved in flight direction (Figure 7b). Reasons for that is the exact determination of the receive time of each pulse as well as the positioning accuracy of the ground receiver in the range of few decimetres. Hence, even small mis-pointing of the antenna could be detected and consequently compensated for.
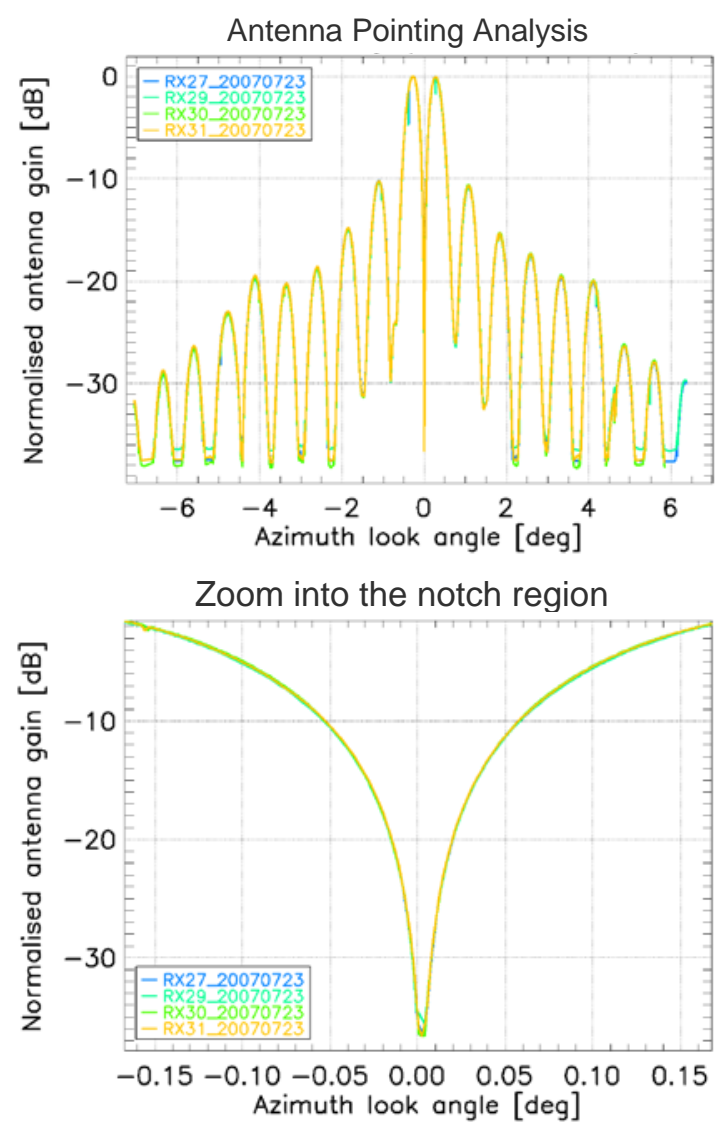

Figure 7a: Azimuth pointing verification

Figure 7b: Zoom into the region around 0 deg look angle.

This was the fact at the beginning of the commissioning phase. A mis-pointing depending on the elevation steering angle was detected. As a mis-pointing in azimuth also means a variation of the Doppler in the SAR raw data, this was detected in the first acquisition processed with the TerraSAR-X Multimode SAR Processor (TMSP) by the TMSP operating team. It could be verified with the pointing measurements.

This is shown in Figure 8. The green line is the dependency derived with dozen of products evaluated by the SAR processor. The orange and red squares define the measurement results of the first reference measurements. The brown tri angles depict the four antenna pointing measurements showing that only four measurements were sufficient to confirm the green curve very well. The blue diamonds finally show the measurements after the correction of the pointing dependency, which was eliminated. The small constant offset was corrected after finalization of the pointing determination corrected which was confirmed by analysis of the SAR products.

The mis-pointing is caused by a rotation of the antenna in its polarisation axis, i. e. a rotation around the antenna's boresight axis. It was corrected, adapting the total-zero Doppler steering law in the on-board tables. A re-verification showed the correct application of the corrections. Long term 
statistics show that there is no residual Doppler offset and hence no mis-pointing remaining.

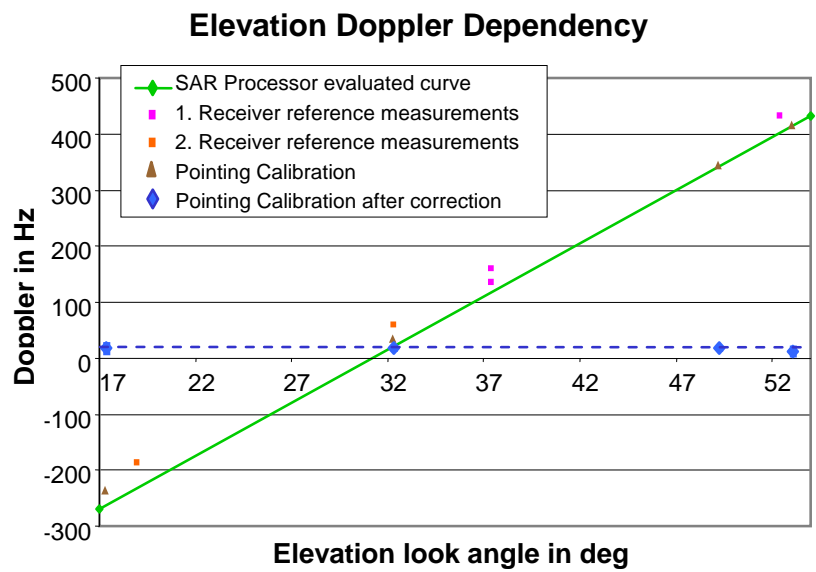

Figure 8: Elevation Doppler Dependency
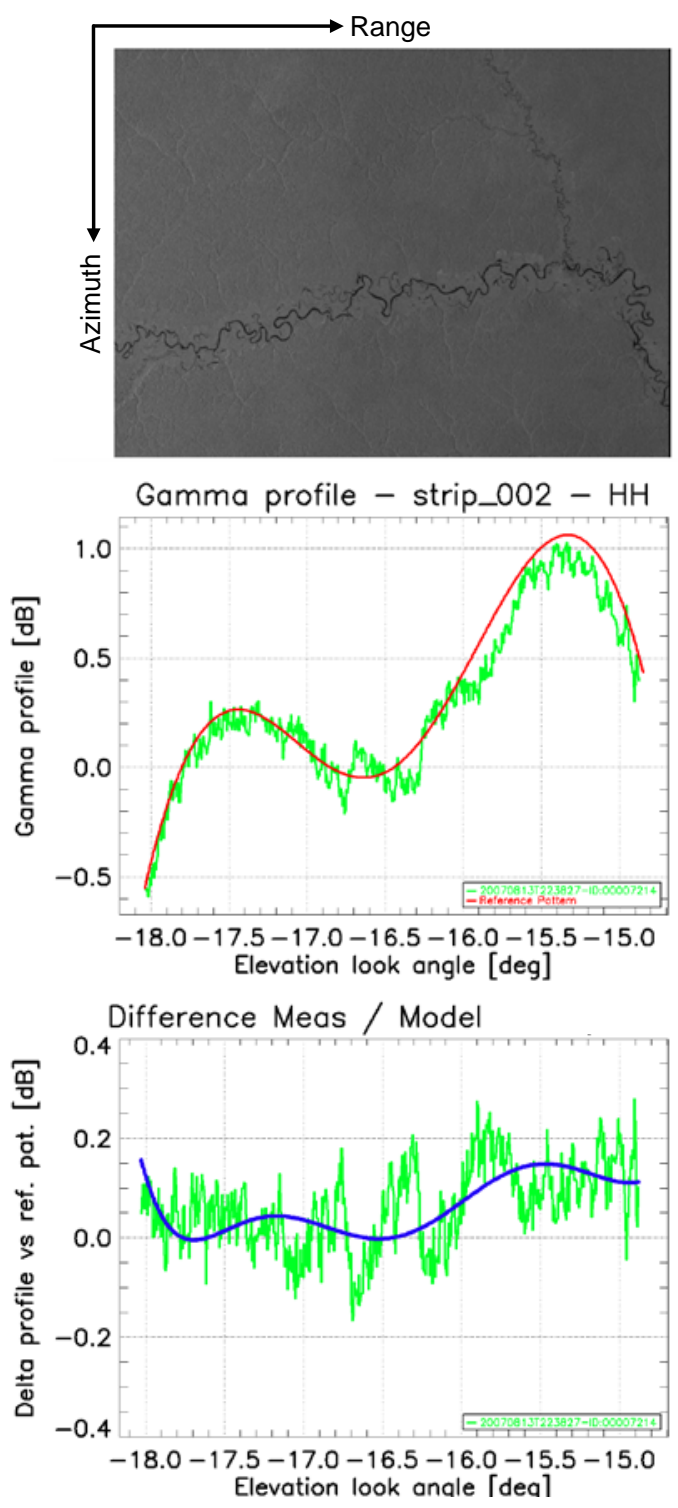

Figure 9a: Amazon rainforest scene used for antenna model verification Figure 9b: Gamma profile of the antenna pattern extracted from rainforest (green curve) and reference pattern (red line) for comparison
Figure 9c: Deviation between gamma profile and reference pattern (green curve) and fit through the deviation (blue line)

\section{Antenna model verification}

\section{1) Antenna model verification in elevation}

After the correct antenna pointing is ensured, the antenna model itself can now be verified. For this purpose, many different acquisitions were evaluated. Generally, these images were four seconds long corresponding to about 12000 azimuth lines.

An exemplary result of the antenna model verification is depicted in Figure 9b. The noisy ripple (in green) is the gamma profile, which now can be compared to the modelled reference pattern depicted in red.

The results show an excellent accordance between the simulated antenna patterns and the measured gamma profiles. This can be seen in Figure 9c, where the deviation between the reference antenna pattern and the estimated pattern is depicted for the exemplary beam. Additionally, by fitting a blue curve into the profile, a noise-freed picture is obtained.

Table 2 summarizes the measurements of the selected beams as well as the maximal deviation between measurement and reference patterns. Several other beams have been measured as well, showing similar performance. Hence, the deviation and consequently the accuracy of the antenna model are within $\pm 0.2 \mathrm{~dB}$ (peak-to-peak) for the pattern shape.

\begin{tabular}{|c|c|c|c|}
\hline Beam & $\begin{array}{c}\text { Antenna } \\
\text { elevation angles }\end{array}$ & $\begin{array}{c}\text { Polari- } \\
\text { sation }\end{array}$ & $\begin{array}{c}\text { Maximal } \\
\text { deviation }\end{array}$ \\
\hline strip_002 & $-18.0^{\circ} . .-14.8^{\circ}$ & $\mathrm{HH}$ & $+0.17 \mathrm{~dB}$ \\
\hline strip_002 & $-18.0^{\circ} . .-14.8^{\circ}$ & $\mathrm{VV}$ & $-0.19 \mathrm{~dB}$ \\
\hline strip_002 & $-18.0^{\circ} . .-14.8^{\circ}$ & $\mathrm{HV}$ & $+0.10 \mathrm{~dB}$ \\
\hline strip_002 & $-18.0^{\circ} . .-14.8^{\circ}$ & $\mathrm{VH}$ & $-0.18 \mathrm{~dB}$ \\
\hline strip_007 & $-6.7^{\circ} . .-4.0^{\circ}$ & $\mathrm{HH}$ & $+0.10 \mathrm{~dB}$ \\
\hline strip_007 & $-6.7^{\circ} . .-4.0^{\circ}$ & $\mathrm{VV}$ & $+0.17 \mathrm{~dB}$ \\
\hline strip_007 & $-6.7^{\circ} . .-4.0^{\circ}$ & $\mathrm{HV}$ & $+0.13 \mathrm{~dB}$ \\
\hline strip_007 & $-6.7^{\circ} . .-4.0^{\circ}$ & $\mathrm{VH}$ & $+0.19 \mathrm{~dB}$ \\
\hline strip_013 & $4.2^{\circ} .6 .1^{\circ}$ & $\mathrm{HH}$ & $+0.08 \mathrm{~dB}$ \\
\hline strip_013 & $4.2^{\circ} . .6 .1^{\circ}$ & $\mathrm{VV}$ & $+0.17 \mathrm{~dB}$ \\
\hline
\end{tabular}

Table 2: Results of the elevation antenna model verification

\section{2) Antenna model verification in azimuth}

As in azimuth direction only the transmit-patterns can be measured with the ground receivers, the accuracy requirement here is half the one as for the two-way patterns, that is $\pm 0.1 \mathrm{~dB}$. With ground receivers it is furthermore possible to verify the side-lobes which is not possible over rainforest. This can be seen in Figure 10a, where even the first grating lobes and 10 side lobes in between are within the measured angular range.

Figure 10a also shows the special case of a double squinted beam nominally only needed for high resolution spotlight acquisitions. In this case a beam steered to angles of $+0.75^{\circ}$ in 
azimuth and $-16.5^{\circ}$ in elevation which is at the specified limits for antenna steering. This beam was specially commanded not to switch the beam during the pass, as in nominal spotlight mode the beam would switch up to 123 times during the acquisition. The measurement results itself consist of about 30000 pulses, where always 10 were averaged to reduce noise.
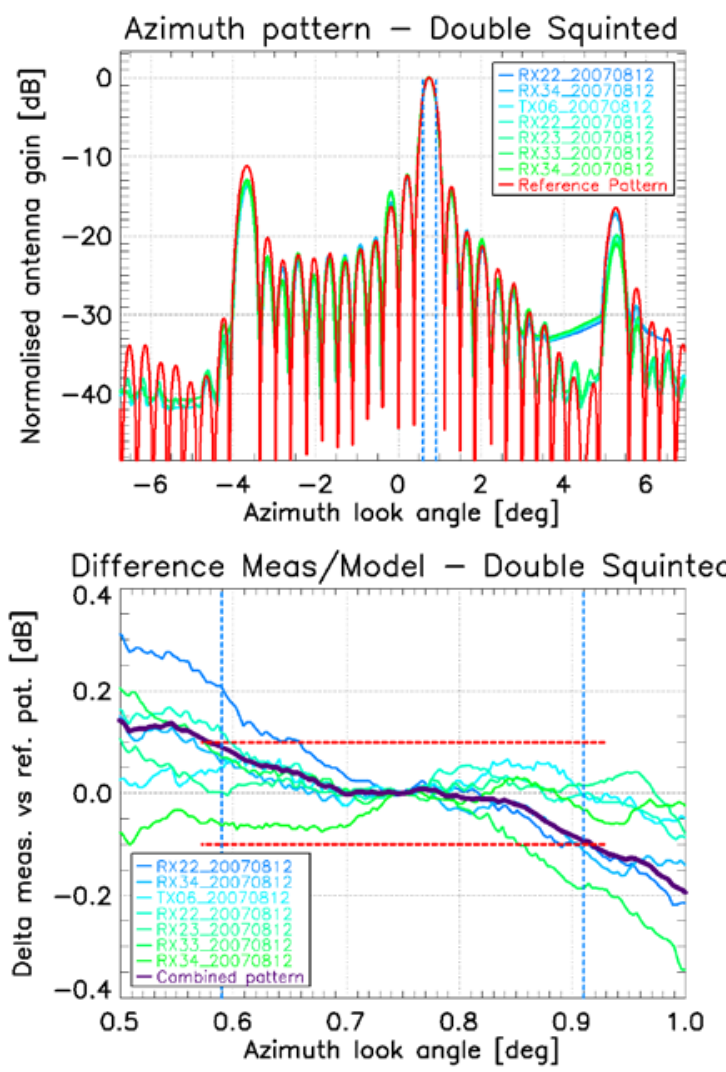

Figure 10a: Transmit patterns compared to a reference pattern, blue to green: measured profiles, red: reference patterns derived by the model

Figure 10b: Deviation between measured and reference pattern.

The antenna patterns measured by ground receivers during one pass are depicted in green to blue and the corresponding reference pattern derived by the model is depicted in red. The resulting deviation between these measurements and the reference pattern is shown in Figure 10b by the purple line, whereby all measurements derived from the ground receiver deployed during one pass were averaged in order to reduce the error contribution of the individual ground receivers.

\begin{tabular}{|l|l|l|}
\hline Beam & $\begin{array}{l}\text { Transmit } \\
\text { polarisatio } \\
\text { n }\end{array}$ & $\begin{array}{l}\text { Max. } \\
\text { Deviation }\end{array}$ \\
\hline strip_002 & H & $+0.09 \mathrm{~dB}$ \\
\hline strip_002 & V & $+0.09 \mathrm{~dB}$ \\
\hline strip_007 & $\mathrm{H}$ & $-0.08 \mathrm{~dB}$ \\
\hline strip_007 & V & $-0.07 \mathrm{~dB}$ \\
\hline strip_013 & $\mathrm{H}$ & $-0.07 \mathrm{~dB}$ \\
\hline strip_013 & V & $-0.08 \mathrm{~dB}$ \\
\hline
\end{tabular}

Table 3: Results of the azimuth antenna model verification
Table 3 summarizes the results for the selected beams of the azimuth verification. As for elevation, the antenna model verification shows extra-ordinary results, i.e. the resulting deviation within the main beam and consequently the accuracy of the model is within the required $\pm 0.1 \mathrm{~dB}$.

The side lobes are also modelled quite well. However, there is no requirement for the accuracy of the side lobes, as they are not used for SAR processing. Only the performance estimation relies on the side lobe modelling for ambiguity ratio calculation. For this task, the accuracy of the close side lobe modelling within $2 \mathrm{~dB}$ is quite sufficient.
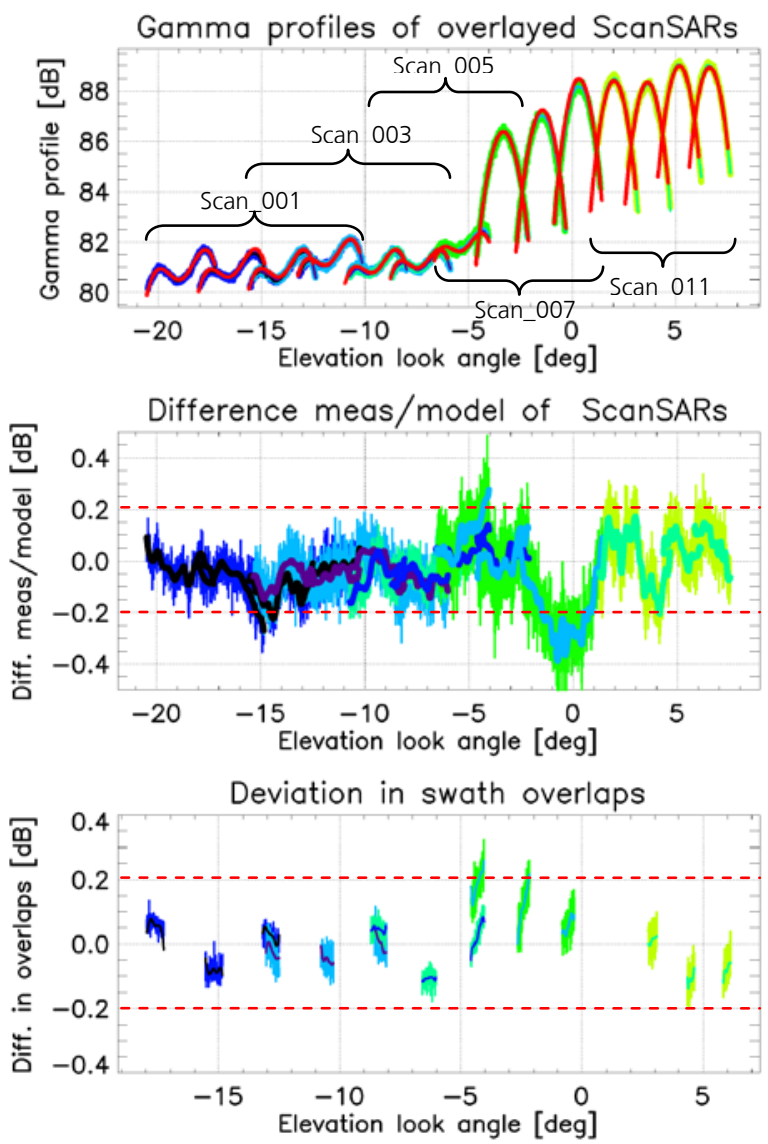

Figure 11a: Verification of the beam-to-beam gain prediction using ScanSAR images, blue to green: measured gamma profiles, red: reference patterns derived by the antenna model.

Figure 11b: Deviation between gamma profile and the reference patterns Figure 11c: Deviation within the overlapping areas of Figure 11b.

\section{3) Verification of the beam-to-beam gain prediction}

Besides the verification of the pattern shape, the capability of the antenna model to predict the difference in the maximum gain between different beams is of great importance. This is intrinsic to the antenna model, as it derives the absolute antenna gain seen from the antenna root point, i. e. the electrical reference point in the SAR instrument. For calibration, this is of great importance: First, it is required for the ScanSAR processing, where four different beams with different antenna gains are acquired, corrected with their corresponding pattern and combined into one image. Second, 
with an appropriate gain prediction, only one absolute calibration factor can be derived for the complete system and not all beams have to be measured during the absolute calibration [9].

The beam-to-beam gain prediction is verified evaluating ScanSAR data. In ScanSAR operation, the beam is switched sequentially between a set of four neighbouring swaths from burst to burst, with one burst lasting for about $0.1 \mathrm{sec}$. This is done to get a broader swath width than for normal Stripmap acquisitions and hence covering about $120 \mathrm{~km}$ in range in contrast to the $30 \mathrm{~km}$ range in Stripmap mode. By generating the un-normalised gamma profile for each of the four swaths, the relative gain deviation between these four swathes can be determined.

In order to obtain the beam-to-beam gain prediction over a wide range of elevation angles including the full performance range (between -15 deg and +9 deg antenna look angle), different sets of ScanSAR acquisitions were combined. The result is shown in Figure 11a. The images were acquired over different parts of the rainforest during different passes. Thus, each illuminated part of the rainforest has different vegetation and consequently different backscatter. Consequently, each complete set of four beams was related to its predecessor set within the overlapping region.
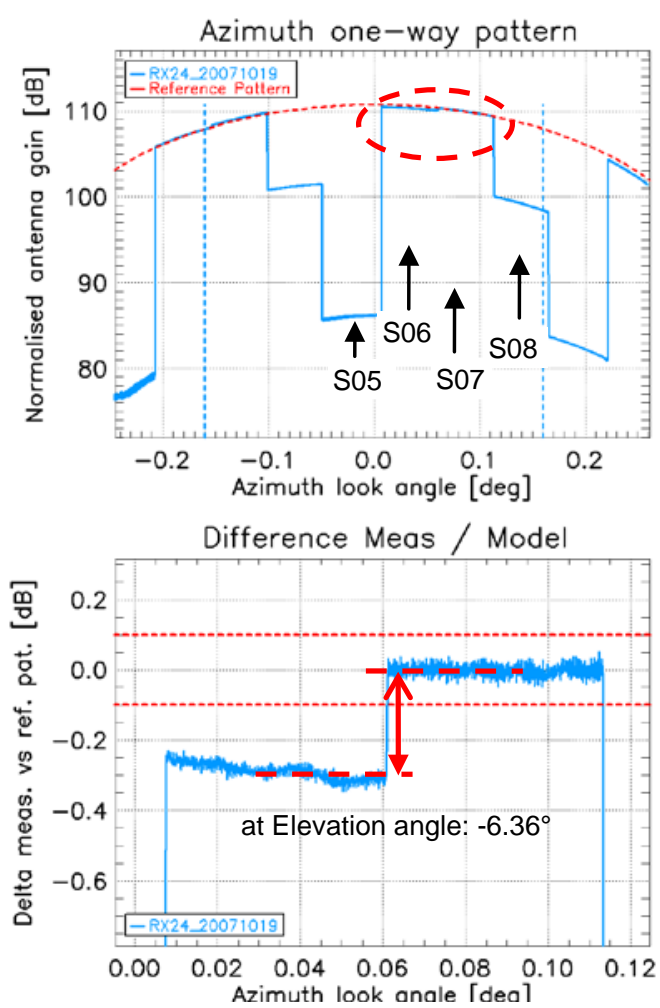

Figure 12a: Verification of the beam-to-beam-gain prediction using ground receivers.

Figure 12b: Zoom into the red-marked area between "s06" and "s07".

Figure 11b shows the deviation between the profiles measured and the corresponding reference pattern. Figure 11c emphasises the deviation between the overlapping regions of two neighbouring swaths. However, the results have an excellent accuracy of below $\pm 0.2 \mathrm{~dB}$ peak-to-peak over the whole angular range and no drift is visible. The slight deviation at about 0 deg elevation angle in Figure 11b results from bad weather conditions disturbing the acquisition. Because illuminating an area of about 560,000 $\mathrm{km}^{2}$ across the rainforest, it is natural to find individual regions with heavy rainfall rates.

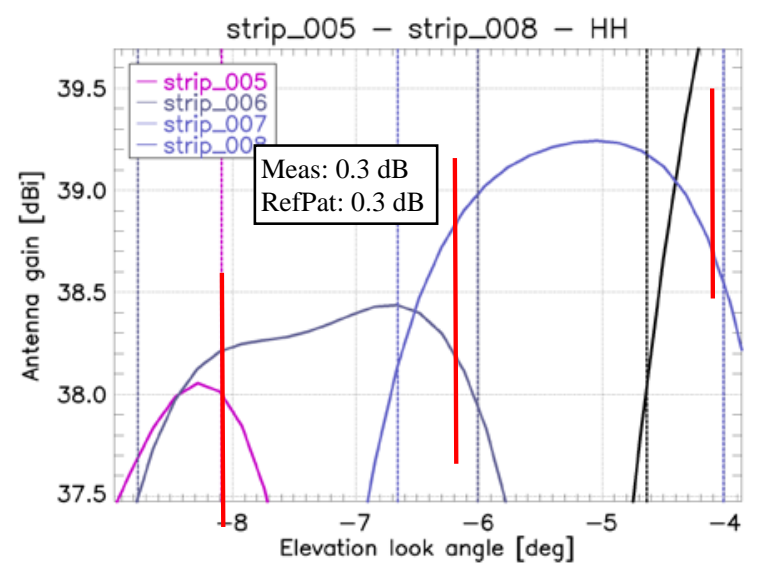

Figure 13: Elevation reference patterns for the corresponding swaths strip_011 to strip_13. For the look angle of the receiver measurement from Figure 12, the deviation between the swaths can be read

A second method to verify the beam-to-beam gain prediction was performed by deploying ground receivers in the overlapping regions of two neighbouring swaths and recording the transmit azimuth patterns. For this purpose the instrument was operated again in ScanSAR mode. As shown in Figure 12a the switching of the instrument between the four beams during one pass is clearly visible in the offsets of the received pulses. The deviation between the overlapping beams can be likewise compared with the antenna model. The corresponding reference antenna patterns generated with the antenna model is shown in Figure 13, where the centre red line corresponds to the elevation angle measured with the ground receiver of Figure 12. In spite of measuring one-way patterns by ground receivers and the interrelated demand on higher accuracy (see above), the maximum deviation is within the required $\pm 0.1 \mathrm{~dB}$ peak-peak.

\section{E. Verification of the cross talk}

Another important aspect for a radar system is the pureness of its polarisations, e. g. a high cross polarisation suppression of the signals meaning minimal cross talk between both polarisations. This is especially important for polarimetric measurements, which can be performed on TerraSAR-X with its dual-polarisation and quad-polarisation modes.

To verify the cross-polarisation suppression, dual polarised data takes were executed and recorded with a ground receiver. The results in Figure 14 show a cross-polarisation suppression of better than $35 \mathrm{~dB}$ for the one-way path, which corresponds to the recommendations of the CEOS SAR Cal/Val Working Group [14]. 


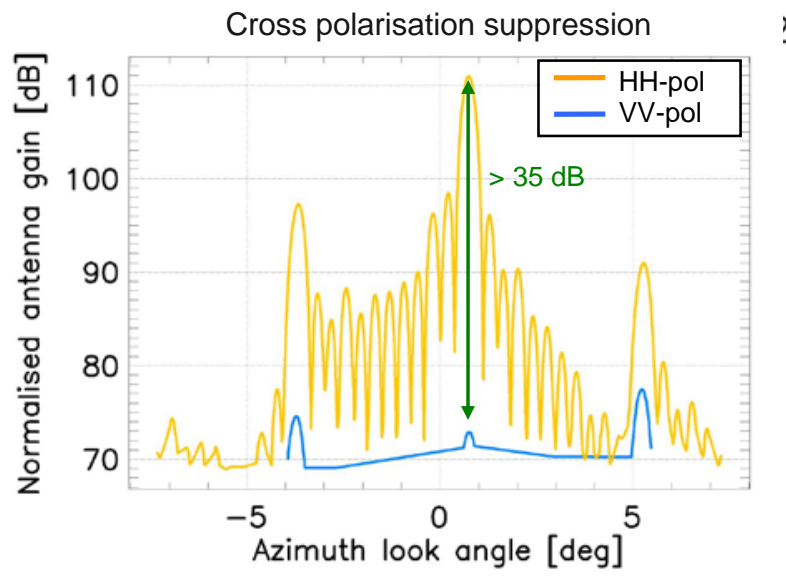

Figure 14: Verification of the cross polarisation suppression.

\section{F. Absolute calibration support}

With the properly working antenna model, the absolute calibration factor required for deriving the radar backscatter coefficient of any target within an image does not have to be measured for all relevant beams. It is measured only for one beam and verified for the same representative beams as used for the antenna model.

By this approach it is possible to shorten the time and the effort extremely for the absolute radiometric calibration performed during the commissioning phase of a SAR system. In case of TerraSAR-X with the verified antenna model, it was sufficient to measure only 3 of the 12000 different beams: one with low, one with mid and one with high incidence angle. The measurement results show an absolute radiometric accuracy of $0.31 \mathrm{~dB}$ (1-sigma) [9].

With the verified antenna model it is furthermore possible to update or change individual beams without performing a dedicated calibration campaign again.

\section{Antenna PATtern MONitoring}

An important task during the operational phase of the TerraSAR-X mission is the continuous monitoring of the antenna patterns, the so called long-term system monitoring (LTSM) of the antenna pattern after completion of the commissioning phase. By this, anomalies in the antenna frontend and especially in the wave guides of the antenna can be detected. Although the instrument is monitored regularly using the PN-Gating method to detect drifted or failed TRMs, the wave guides are not covered by PN-Gating. Hence, they have to be monitored separately using external targets like the rainforest.

By acquiring images over the Amazon rainforest regularly once per cycle, the reference antenna patterns can be compared to the derived patterns of the individual acquisitions as described before. This is done for the ScanSAR-Beam scan_003, which beams are tapered and therefore should be affected by deformations more than untapered beams.
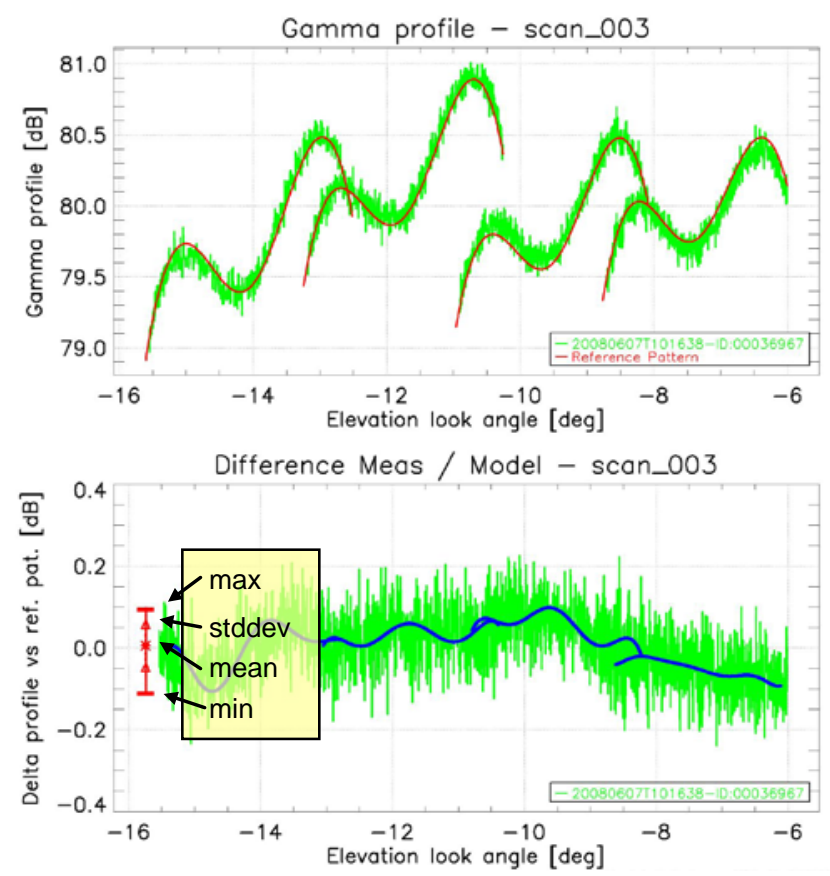

Figure 15a: ScanSAR acquisition of the rainforest

Figure 15b: Quality parameter derivation for LTSM

After calculating the difference between measurement and the reference pattern and fitting through the deviation, three quality parameters can be derived as depicted in Figure 15b:

- the mean pattern deviation,

- its standard deviation and

- its maximal/minimal deviation

Especially the maximal/minimal deviation is a good measure for the quality of the antenna patterns. It should stay within the range of $\pm 0.2 \mathrm{~dB}$. Figure 16 shows the quality parameters for each acquired LTSM data take over time, the values are referenced to their individual mean value.

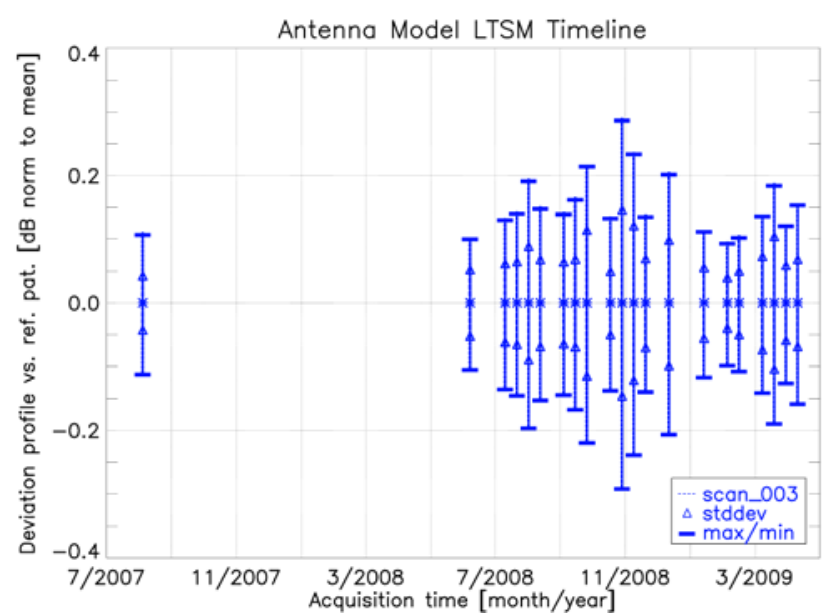

Figure 16: Relative deviation between measured pattern and model over time

To gather knowledge about the absolute variation of the antenna gain, the measurements were referenced to the first measurement acquired during the TerraSAR-X Commissioning Phase in August 2007. Here in Figure 17, a 
cyclic variation can be seen. This variation was also recognized in L-Band and C-Band images over rainforest, e. g. for RadarSAT-1 [10] where it evolves sinusoidal. The variation is caused by seasonal effects of the vegetation in the Amazon rainforest.

In X-band the variation of $\pm 0.8 \mathrm{~dB}$ seems to be little higher than in C-Band with $\pm 0.5 \mathrm{~dB}$. However, the temporal devolution is in accordance with the C-Band measurements.

To exclude an effect of the instrument, the Absolute Calibration Factor was verified over ground targets, showing the stability within the measurement accuracy [11].

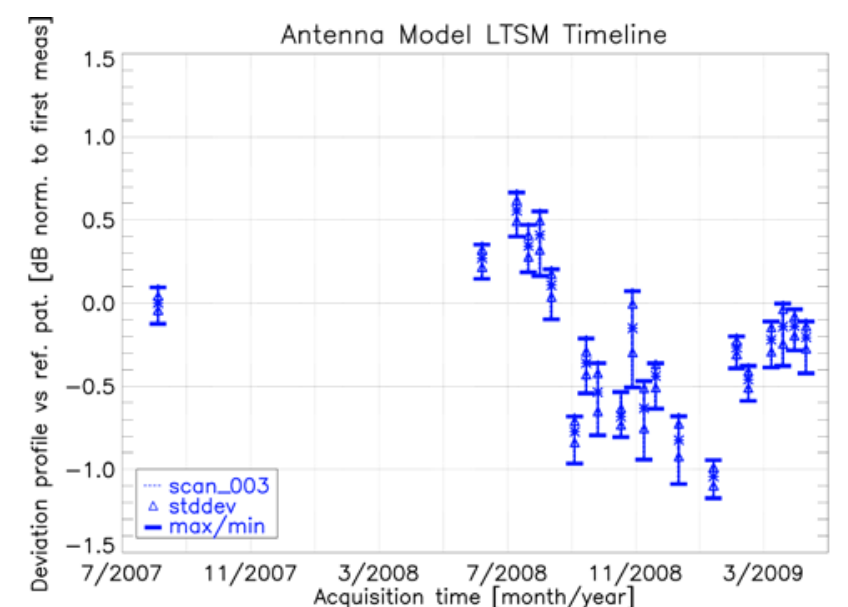

Figure 17: Absolute deviation between measured pattern and model over time

\section{VIII.CONCLUSION}

The TerraSAR-X antenna model is utilised for generating the reference antenna patterns in order to correct the antenna characteristics in the SAR images during SAR processing. Furthermore is enables beam optimisation to derive optimal beam settings or re-optimisation in case of contingencies. A high accuracy of the antenna model is achieved by precise onground measurements of the embedded subarray patterns as well as a highly stable internal calibration of the instrument. To ensure the specified product quality, its accuracy has been verified in orbit during the commissioning phase following the TerraSAR-X launch in June 2007. The results show the excellent accuracy of both, the whole TerraSAR-X system itself and the antenna model.

At first, the pointing of the system was determined with accuracies of better than 0.008 deg in elevation and better than 0.002 deg in azimuth direction. The antenna model itself was verified in elevation using Amazon rainforest on providing the reference antenna patterns with an accuracy of better than $\pm 0.2 \mathrm{~dB}$ (peak-to-peak). In azimuth, where transmit-pattern were determined with ground receivers, an accuracy of $\pm 0.1 \mathrm{~dB}$ (peak-to-peak) was achieved. The prediction of the beam-to-beam gain offset could also be verified with $\pm 0.2 \mathrm{~dB}$ (peak-to-peak) accuracy enabling accurate image referencing, a short commissioning phase and the flexibility to update beams without the necessity of a dedicated calibration campaign.
The long-term stability of the antenna patterns is performed during the operational phase. After 1.5 years of operation it shows the antenna patterns still staying within $\pm 0.2 \mathrm{~dB}$.

The excellent results of TerraSAR-X show, that the antenna model approach was successfully applied and the approach can be used for future system like TanDEM-X.

\section{ACKNOWLEDGEMENT}

A great thank is dedicated to the whole TerraSAR-X team and especially to the HR calibration team for supporting the antenna model verification with an uncountable number of calibration campaigns and their unweary effort at every possible weather and environmental conditions.

Furthermore a special thank to the Astrium GmbH for performing the highly accurate embedded antenna pattern measurements and on-ground validation and developing the TerraSAR-X as such a highly accurate SAR system.

\section{REFERENCES}

[1] Manfred Zink, Betlem Rosich: Antenna Elevation Pattern Estimation from Rain Forest Acquisitions, ENVISAT/ASAR Calibration Review (ECR) of ESTEC, European Space Agency (ESA), 2002, Noordwijk, Netherlands

[2] S. Buckreuss, R. Werninghaus, W. Pitz: German Satellite Mission TerraSAR-X; 2008 IEEE Radar Conference, Rome, Italy, 2008

[3] B. Grafmüller, A. Herschlein, C. Fischer: The TerraSAR-X Antenna System; Radar Conference, IEEE International, May 2005

[4] B. Bräutigam, J. Hueso González, M. Schwerdt, M. Bachmann: Radar Instrument Calibration of TerraSAR-X, 7th European Conference on Synthetic Aperture Radar, Friedrichshafen, Germany, June 2008

[5] M. Bachmann, M. Schwerdt, B. Bräutigam, B. Grafmüller, A. Herschlein, J. L. Álvarez-Pérez: The TerraSAR-X Antenna Model Approach; Proceedings on INICA 07, Munich, Germany, March 2007

[6] J. L. Álvarez-Pèrez, M. Schwerdt, M. Bachmann: TerraSAR-X Antenna Pattern Estimation by Complex Treatment of Rain Forest Measurements; Geoscience and Remote Sensing Symposium, IGARSS 2006, Denver, USA, July 2006

[7] M. Schwerdt, D. Hounam, J. L. Alvarez-Pèrez: The calibration concept of TerraSAR-X: a multiple-mode, high-resolution SAR, Canadian Journal of Remote Sensing, Vol. 31, No. 1, pp. 30-36, 2005

[8] J. E. Laycock, H. Laur: ERS-1 SAR Antenna Pattern Estimation ES-TN-DPE-OM-JL01, iss. 1, rev. 1, September 1994, http://earth.esa.int/ers/instruments/sar/sar_AP_est/

[9] M. Schwerdt, B. Bräutigam, M. Bachmann, B. Döring, J. HuesoGonzalez, D. Schrank: Final Results of the Efficient TerraSAR-X Calibration Method, IEEE Radar Conference, Rome, Italy, May 2008

[10] B. Döring, M. Schwerdt, R. Bauer: TerraSAR-X Calibration Ground Equipment, Proceedings of WFMN07, Chemnitz, Germany, 2007

[11] A. P. Luscombe: RadarSAT-2 Calibration: Proposed Targets and Techniques, IEEE Paper, 2001, 0-7803-7031-7

[12] C. A. Balanis: Antenna Theory: Analysis and Design, 2nd ed. John Wiley and Sons, 1997.

[13] B. Bräutigam, M. Schwerdt, M. Bachmann: An Efficient Method for Performance Monitoring of Active Phased Array Antennas, IEEE Transactions on Geoscience and Remote Sensing (TGRS), Vol. 47, No. 4, April 2009, pp. 1236-1243, ISSN 0196-2892

[14] Recommendations from the CEOS SAR CAL/VAL 2004, ESA Earthnet online, Available: http://earth.esa.int/workshops/ ceos_sar_2004/recommendation_at_ceos_cal.htm, 2009-01-30

[15] A. Meta, J. Mittermayer, U. Steinbrecher, P. Prats: Investigations on the TOPSAR acquisition mode with TerraSAR-X. Proceedings of IEEE Geoscience and Remote Sensing Symposium (IGARSS), 2007, Barcelona, Spain 